\title{
ARTICLE
}

Received 22 Jan 2014 | Accepted 7 Mar 2014 | Published 8 Apr $2014 \quad$ DOl: 10.1038/ncomms4586

\section{Excitons versus free charges in organo-lead tri-halide perovskites}

\author{
Valerio D'Innocenzo ${ }^{1,2, \star}$, Giulia Grancini ${ }^{1, \star}$, Marcelo J.P. Alcocer ${ }^{1,2}$, Ajay Ram Srimath Kandada ${ }^{1}$ \\ Samuel D. Stranks ${ }^{3}$, Michael M. Lee ${ }^{3}$, Guglielmo Lanzani ${ }^{1,2}$, Henry J. Snaith ${ }^{3}$ \& Annamaria Petrozza ${ }^{1}$
}

Excitonic solar cells, within which bound electron-hole pairs have a central role in energy harvesting, have represented a hot field of research over the last two decades due to the compelling prospect of low-cost solar energy. However, in such cells, exciton dissociation and charge collection occur with significant losses in energy, essentially due to poor charge screening. Organic-inorganic perovskites show promise for overcoming such limitations. Here, we use optical spectroscopy to estimate the exciton binding energy in the mixed-halide crystal to be in the range of $50 \mathrm{meV}$. We show that such a value is consistent with almost full ionization of the exciton population under photovoltaic cell operating conditions. However, increasing the total photoexcitation density, excitonic species become dominant, widening the perspective of this material for a host of optoelectronic applications.

\footnotetext{
${ }^{1}$ Center for Nano Science and Technology@PoliMi, Istituto Italiano di Tecnologia, via Giovanni Pascoli 70/3, Milan 20133, Italy. ${ }^{2}$ Dipartimento di Fisica, Politecnico di Milano, Piazza L. da Vinci, 32, Milano 20133, Italy. ${ }^{3}$ University of Oxford, Clarendon Laboratory, Parks Road, Oxford OX1 3PU, UK. *These authors contributed equally to this work. Correspondence and requests for materials should be addressed to H.J.S. (email: h.snaith1@physics.ox.ac.uk) or to A.P. (email: annamaria.petrozza@iit.it).
} 
P erovskite solar cells were first introduced in a dye-sensitized configuration, where the dye was replaced by organo-lead halide perovskite crystals (e.g. $\mathrm{CH}_{3} \mathrm{NH}_{3} \mathrm{PbI}_{3}$ or $\mathrm{CH}_{3} \mathrm{NH}_{3} \mathrm{PbI}_{3-} \mathrm{Cl}_{\mathrm{x}}$ ) sensitizing the surface of mesoporous $\mathrm{TiO}_{2}$ (refs 1-5). In the perovskite-sensitized configuration, developed within the excitonic solar cell concept, there is no requirement for long-range exciton or charge diffusion, as the perovskite crystals are sandwiched within a nanoscale heterojunction between an electron-accepting metal oxide $\left(\mathrm{TiO}_{2}\right)$ and a hole-accepting organic hole-transporter (or redox active electrolyte) and longrange transport occurs in those phases. Although the rapid advancement of this sensitized approach has occurred, there has been in parallel a striking divergence in technology, where the mesoporous $\mathrm{TiO}_{2}$ has been proven non-essential ${ }^{5}$, and simply a solid absorber layer sandwiched within a planar heterojunction has been demonstrated to operate extremely well, with powerconversion efficiencies of over $15 \%$ already reported ${ }^{6,7}$. The perovskite absorbers thus appear capable of operating in a similar manner to excitonic absorbers, but also in a comparable configuration and with comparable performance to the best inorganic thin-film semiconductors ${ }^{6,8}$. The fundamental question that arises from these studies concerns the nature of the elementary photoexcitations that are involved in the photovoltaic (PV) mechanisms; in particular whether bound excitons are created and transport energy to the heterojunction, thus requiring further ionization at the heterojunction, or whether free charges are spontaneously generated within the bulk of the perovskite ${ }^{9-12}$. In order to answer this question, we explicitly need to know the exciton binding energy in the room temperature (RT) crystal phase of the perovskite absorber, and to understand the branching ratio between free carriers and excitons under operating solar cell conditions.

In this work, we use the temperature dependence of the absorption band edge to estimate the exciton binding energy in $\mathrm{CH}_{3} \mathrm{NH}_{3} \mathrm{PbI}_{3-\mathrm{x}} \mathrm{Cl}_{\mathrm{x}}$ perovskite crystals. Subsequently, we use this value in the Saha equation, which governs ionization processes $^{13,14}$, to estimate the equilibrium branching ratio between free charges and bound excitons as a function of temperature and charge density. We find that at equilibrium, following photoexcitation, there is a predominant fraction, near unity, of free charges in the PV operating regime.

\section{Results}

Temperature dependence of optical absorption. In Fig. 1, we show the optical absorption spectra taken in a broad temperature $(T)$ range from $\mathrm{RT}=290 \mathrm{~K}$ to $4.2 \mathrm{~K}$, on a polycrystalline film of $\mathrm{CH}_{3} \mathrm{NH}_{3} \mathrm{PbI}_{3}$ and of its mixed-halide analogue $\mathrm{CH}_{3} \mathrm{NH}_{3} \mathrm{PbI}_{3-x} \mathrm{Cl}_{x}$. We study both flat films deposited on glass, which are polycrystalline with crystal sizes larger than $500 \mathrm{~nm}$ in diameter ${ }^{7}$ (Fig. 1a,b), and perovskite films infiltrated into a mesoporous oxide scaffold, where the perovskite crystals are on the order of 60 to $100 \mathrm{~nm}$ in size ${ }^{7}$ (Fig. 1c,d). Figure 1a shows the spectra of the $\mathrm{CH}_{3} \mathrm{NH}_{3} \mathrm{PbI}_{3}$ in a spectral range between $690 \mathrm{~nm}$ and $825 \mathrm{~nm}$. A sharp peak at $\sim 765 \mathrm{~nm}$ emerges at lower temperatures, which we attribute to an excitonic transition. At $170 \mathrm{~K}$, a second excitonic peak emerges at $740 \mathrm{~nm}$. Upon further reduction of temperature, the latter peak gains strength in intensity and monotonically shifts to longer wavelengths, whereas the first peak disappears. We assign this behaviour to a phase transition of the perovskite crystal, notably from a tetragonal to an orthorhombic structure ${ }^{15,16}$, which commences at $170 \mathrm{~K}$, inducing a blue shift in the absorption edge of about $60 \mathrm{meV}$, and completes at $150 \mathrm{~K}$. Within this temperature range, the two phases coexist, possibly due to the polycrystalline nature of the sample. At $4.2 \mathrm{~K}$ a single excitonic feature peaks at $\sim 760 \mathrm{~nm}$, similar to what has been observed in single crystals of $\mathrm{CH}_{3} \mathrm{NH}_{3} \mathrm{PbI}_{3}$ (refs 17,18).

At RT, however, we cannot observe a clear gap between the excitonic peak and the conduction band continuum. We also note that as the temperature increases, especially in the lowtemperature stable crystalline phase, the absorption band edge blue shifts. This 'Varshni' trend is often observed in lead composite semiconductors with a positive thermal expansion coefficient of the band gap, $\mathrm{d} E_{\mathrm{g}} / \mathrm{d} T^{19,20}$.

The $\mathrm{CH}_{3} \mathrm{NH}_{3} \mathrm{PbI}_{3-x} \mathrm{Cl}_{x}$ perovskite absorption spectra are presented in Fig. 1b. They show a similar trend in temperature, with evident positive $\mathrm{d} E_{\mathrm{g}} / \mathrm{d} T$ in the low-temperature phase; however, some important differences exist. First of all, the phase transition is activated at a lower temperature $(\sim 140 \mathrm{~K})$, and is completed by $120 \mathrm{~K}$, probably due to different chemical/structural environments in the two compounds. Secondly, the excitonic peak emerges with a stronger intensity with respect to the broad continuum (see spectrum at $4.2 \mathrm{~K}$ ).

A different scenario appears when looking at the absorption spectra of the perovskite crystallites formed in the mesoporous $\mathrm{Al}_{2} \mathrm{O}_{3}$ scaffold (Fig. 1c,d), which imposes a smaller crystal size of the crystals ${ }^{7}$. Both materials show a broad band edge with no evident excitonic peak and are blue shifted with respect to the flat samples. The structural phase transition can still be observed at the same temperature for the $\mathrm{CH}_{3} \mathrm{NH}_{3} \mathrm{PbI}_{3-x} \mathrm{Cl}_{x}$ (see Supplementary Fig. 1 in the SI where the complete temperature range is reported). However, upon cooling, the spectra remain featureless. Thus, smaller crystals show virtually no resolvable excitonic transition. We can exclude any confinement phenomena as in this case one would expect an opposite trend, that is, appearance of the exciton optical transition as the crystal size is reduced. On the other hand, the reduction in size, the enhancement of the surface area and the presence of a scaffold during the crystallization process is likely to increase the relative contribution of surface effects, which may result in structural strains and/or doping mechanisms, which notably reduce the exciton binding energy ${ }^{21}$.

Interestingly, when we fabricate a combination of the above sample structures, that is, a thin $\sim 80 \mathrm{~nm}$ interpenetrated mesostructured composite covered by a $\sim 350 \mathrm{~nm}$ flat perovskite capping layer (see cartoon in Fig. 2a), as is the case for the optimized device presented in ref. 7 , the resulting absorption spectrum is a combination of the absorption spectra of the two layers (Fig. 2b). This suggests that the presence of the thin mesoporous interlayer is responsible for tuning the optoelectronic properties of the material by tuning the crystal size. This may have important consequences for device performance, where generally higher photovoltages are obtained for mesostructured devices than for planar structures ${ }^{7}$.

Exciton binding energy estimation. The optical analysis presented above suggests the presence of an excitonic transition, probably very close to the continuum. Indeed, the presence of a Wannier-Mott exciton with a binding energy of about $45 \mathrm{meV}$ has been predicted in the single crystal of $\mathrm{CH}_{3} \mathrm{NH}_{3} \mathrm{PbI}_{3}$ at low temperatures ${ }^{17,18}$. Temperature-dependent, time-resolved photoluminescence (PL) is one approach for estimating the exciton binding energy in a semiconductor. We indeed observed a reduction in $\mathrm{PL}$ intensity of $\mathrm{CH}_{3} \mathrm{NH}_{3} \mathrm{PbI}_{3-x} \mathrm{Cl}_{x}$ when increasing the temperature; however, the PL dynamics did not allow for an unambiguous assignment to the enhancement in thermal dissociation rate of excitons at higher temperatures. As such, here, we estimate an upper limit of the exciton binding energy in $\mathrm{CH}_{3} \mathrm{NH}_{3} \mathrm{PbI}_{3-x} \mathrm{Cl}_{x}$, by using the temperature-dependent absorption line width of the observed excitonic transition. 
a

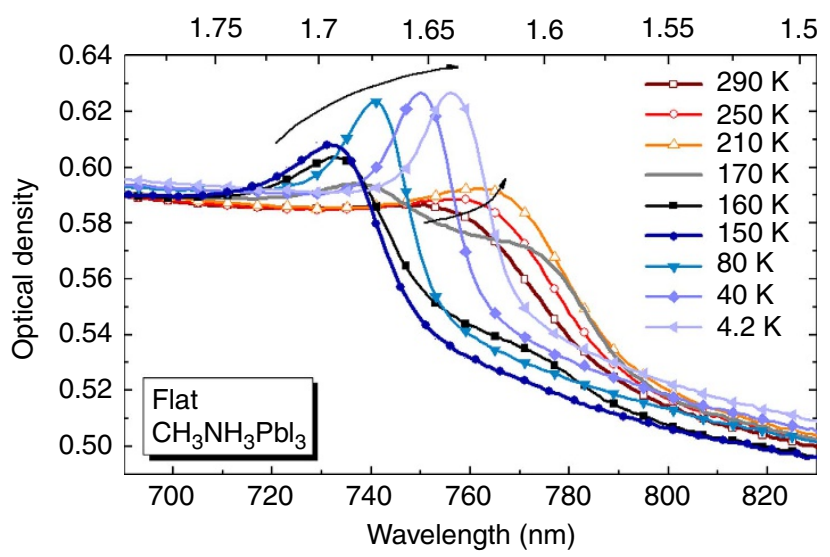

C

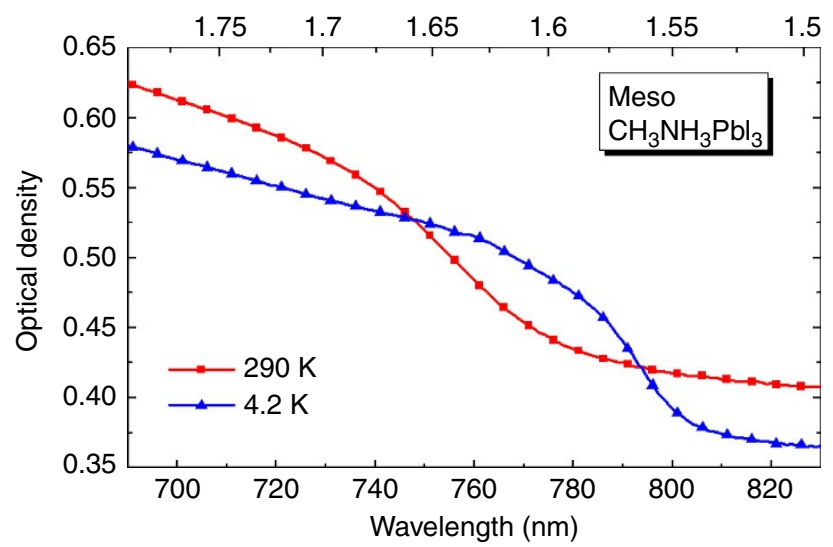

b

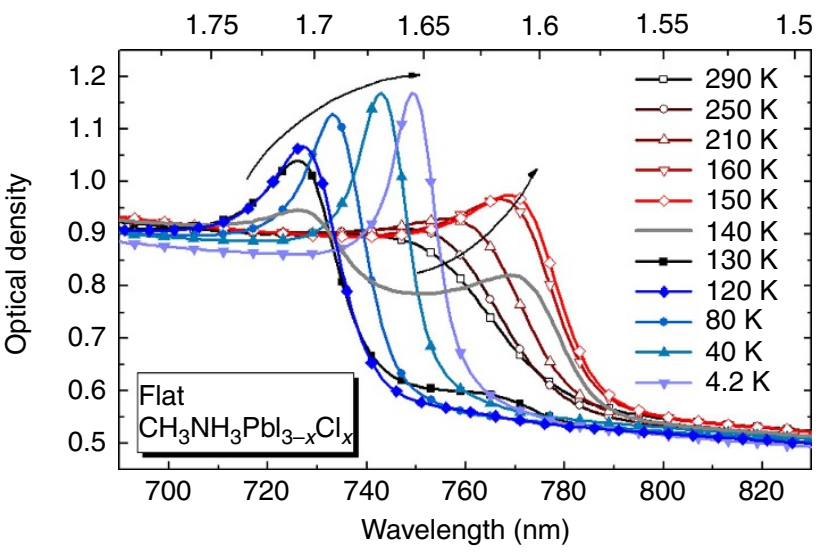

d

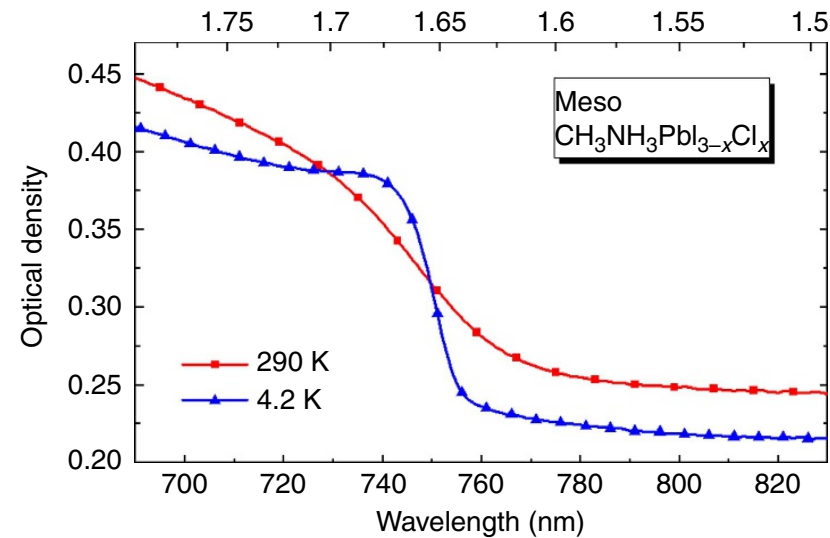

Figure 1 | Temperature dependence of the absorption spectra of the mixed-halide perovskites. Absorption spectra of $\mathrm{CH}_{3} \mathrm{NH}_{3} \mathrm{Pbl}_{3}$ and $\mathrm{CH}_{3} \mathrm{NH}_{3} \mathrm{Pbl}_{3-x} \mathrm{Cl}_{x}$ as a function of temperature $(290-4.2 \mathrm{~K})$. (a, b) Thin films on a glass substrate and (c, d) on a mesoporous $\mathrm{Al}_{2} \mathrm{O}_{3}$ substrate.

a

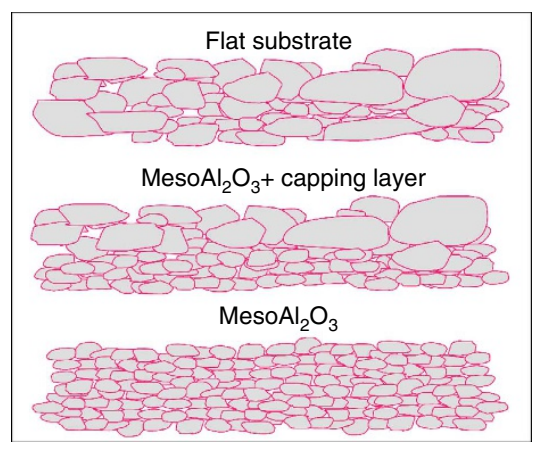

b

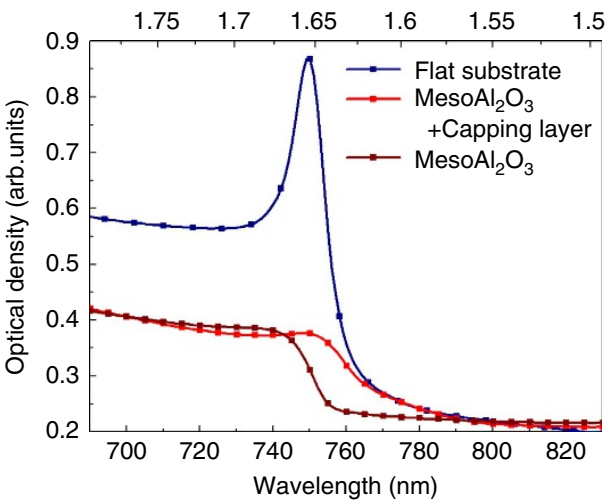

Figure 2 | Absorption spectra of mesostructured thin films. (a) Schematic presentation of the average crystals dimension for differently prepared samples, that is, deposited on a glass substrate (top), on a thick $\mathrm{Al}_{2} \mathrm{O}_{3}$ scaffold without a perovskite capping layer (bottom) and a thin $\mathrm{Al}_{2} \mathrm{O}_{3}$ scaffold with a perovskite capping layer (middle). (b) Absorption spectra of $\mathrm{CH}_{3} \mathrm{NH}_{3} \mathrm{Pbl}_{3}{ }_{-x} \mathrm{Cl}_{x}$, thin films collected at $4.2 \mathrm{~K}$. The films were deposited on a flat glass substrate (blue line), a thick mesoporous $\mathrm{Al}_{2} \mathrm{O}_{3}$ (dark red line) and according to the optimized device structure where part of the perovskite film is infiltrated in the mesoporous $\mathrm{Al}_{2} \mathrm{O}_{3}$ and an additional crystalline perovskite capping layer is present ${ }^{7}$ (red line). For better comparison, spectra are offset.

As inhomogeneous broadening due to disorder is generally temperature independent, here we only take into account the homogeneous broadening mechanism within the two-level system $^{22}$. The coherence lifetime $T_{2}$ of the excited state transition can be affected by the natural population decay of the excited state $\left(T_{1}\right)$ and by elastic scattering events (with rate $\gamma$ ) that do not change the excited state population but contribute to the broadening of the transition. For most polar inorganic solids at high temperatures (for example, $T>100 \mathrm{~K}$ ), phonon interaction leads predominantly to exciton ionization (through inelastic 
scattering $)^{22,23}$. This approximation is valid in our case, as the exciton absorption broadening occurs together with a reduction in the intensity of the optical excitonic transition, thus the population decay rate, $1 / T_{1}$, can be described as the sum of an intrinsic decay rate $\left(k_{0}\right)$ and thermal dissociation rate $\left(k_{\mathrm{T}}\right)^{22}$,

$$
\frac{1}{T_{1}}=k_{0}+k_{\mathrm{T}} \text {. }
$$

We model the rate of exciton thermal dissociation $\left(k_{\mathrm{T}}\right)$ according to the following equation,

$$
k_{\mathrm{T}}=v_{\mathrm{T}} e^{-E_{\mathrm{B}} / k_{\mathrm{B}} T}
$$

where $E_{\mathrm{B}}$ is the exciton binding energy, $k_{\mathrm{B}}$ is the Boltzmann constant and $v_{\mathrm{T}}$ is the attempt frequency. Substituting the relationship between $T_{1}$ and $T_{2}, \frac{1}{T_{2}}=\frac{1}{2 T_{1}}+\gamma$ (ref. 22), into the

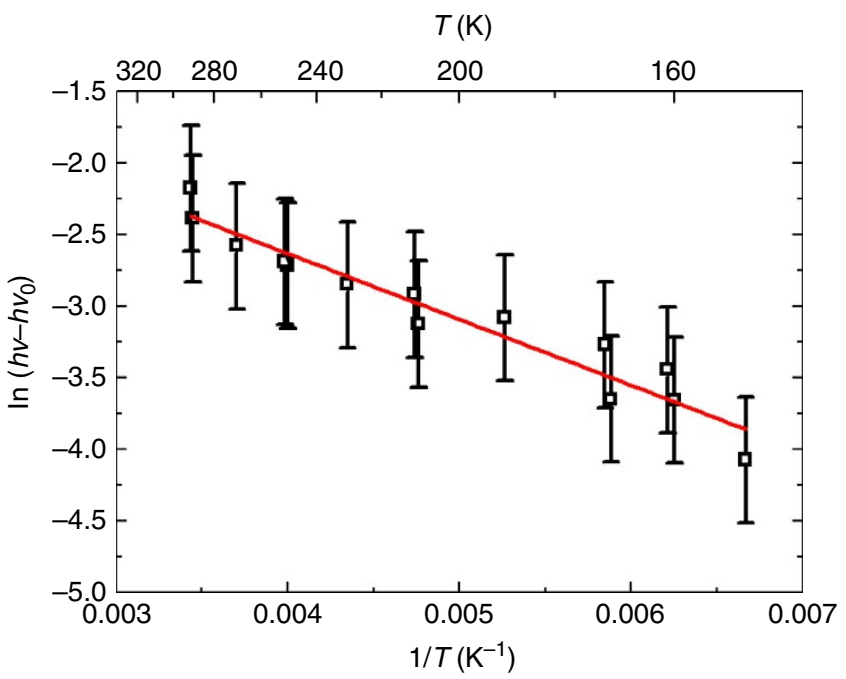

Figure 3 | Estimation of the exciton binding energy. Plot of the exciton absorption band broadening $h \Delta v$ in $\mathrm{CH}_{3} \mathrm{NH}_{3} \mathrm{Pbl}_{3}{ }_{-} \mathrm{Cl}_{x}$ flat layer vs inverse temperature $(150 \mathrm{~K}<\mathrm{T}<290 \mathrm{~K})$. For each point, the error bar indicates the maximum variation of the experimental value due to the $\pm 10 \mathrm{~K}$ uncertainty in the determination of the sample temperature. The solid line is the fit realized according to equation (4), which gives an estimation for the binding energy of $E_{\mathrm{B}}=(55 \pm 20) \mathrm{meV}$ and an attempt frequency of $(7 \mathrm{fs})^{-1}$. $\chi^{2}=0.92$.

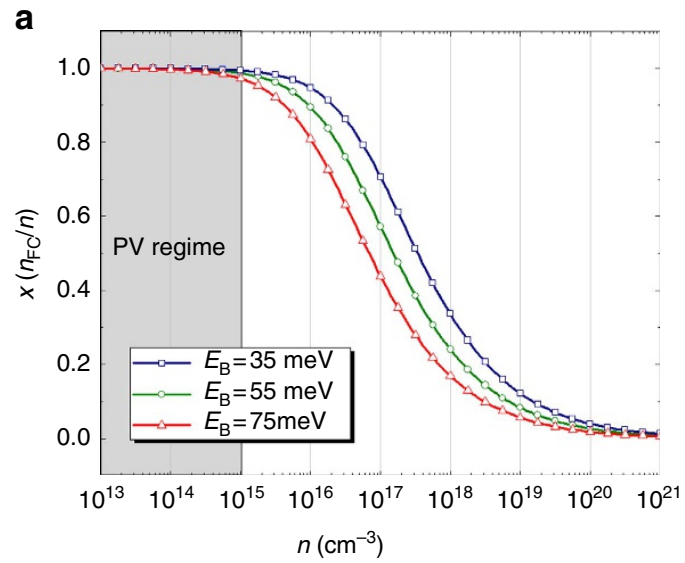

equation for the line width, $\Delta v=1 / \pi T_{2}$ we obtain:

$$
\Delta v=\Delta v_{0}+v_{\mathrm{T}} e^{-E_{\mathrm{B}} / k_{\mathrm{B}} T}
$$

where $\Delta v_{0}$ accounts for the temperature-independent broadening. For a better fit of the temperature dependence of the experimental line width $h \Delta v$, the linear form of equation (3) is considered:

$$
\ln \left(h \Delta v-h \Delta v_{0}\right)=\ln \left(h v_{\mathrm{T}}\right)-E_{\mathrm{B}} / k_{\mathrm{B}} T
$$

In Fig. 3, the natural logarithm of the excitonic transition line width of $\mathrm{CH}_{3} \mathrm{NH}_{3} \mathrm{PbI}_{3-} \mathrm{Cl}_{x}$ is plotted as a function of the inverse of temperature in the range between $150 \mathrm{~K}$ and $290 \mathrm{~K}$. The data are visualized in such a scale to emphasize the match between the experimental trend and the theoretical model. By fitting the data with equation (4), (Fig. 3), we estimate an exciton binding energy of $55 \pm 20 \mathrm{meV}$. The fits are shown in Fig. 3 along with the experimental data. For statistical relevance, the points reported in the graph are derived from repeated measurements of many different samples. Details of the analysis are presented in section Supplementary Methods of the SI.

Numerical simulation of the fraction of free charges vs excitons. Under thermodynamic equilibrium, there is a coexistence of free charges and excitons, and an interchange between the two species. The fraction of free charges $\left(n_{\mathrm{FC}}\right)$ vs. excitons $\left(n_{\text {exc }}\right)$ is not only determined by the binding energy, which determines how long electron-hole pairs remain bound, but also by the excitation density $(n)$, which influences how often electrons and holes meet to form excitons ${ }^{13,14}$.

In the PV working regime, the total excitation density can be estimated from the absorbed solar flux and the excitation lifetime. We can estimate the absorbed solar flux by integrating the absorption of the perovskite film over the $100 \mathrm{mWcm}^{-2}$ AM1.5 solar spectrum. The species lifetime can be estimated by measuring the PL decay in neat perovskite films (equivalent to operation under open-circuit conditions with ideal n- and p-type contacts that introduce no additional non-radiative decay channels) or in perovskite films contacted by a selective holeor electron acceptor (similar to the scenario in the operating solar cells), which we have measured to be $\sim 270 \mathrm{~ns}$ and $5 \mathrm{~ns}$, respectively ${ }^{24}$. We hence determine the steady state excitation density (where excitations can be free charge or excitons) to be between $10^{13}$ and $10^{15} \mathrm{~cm}^{-3}$. If we assume a dielectric constant of 6.5 (ref. 18) and a binding energy of approximately $50 \mathrm{meV}$, the exciton radius can be calculated to be $\sim 2.3 \mathrm{~nm}$. Considering this

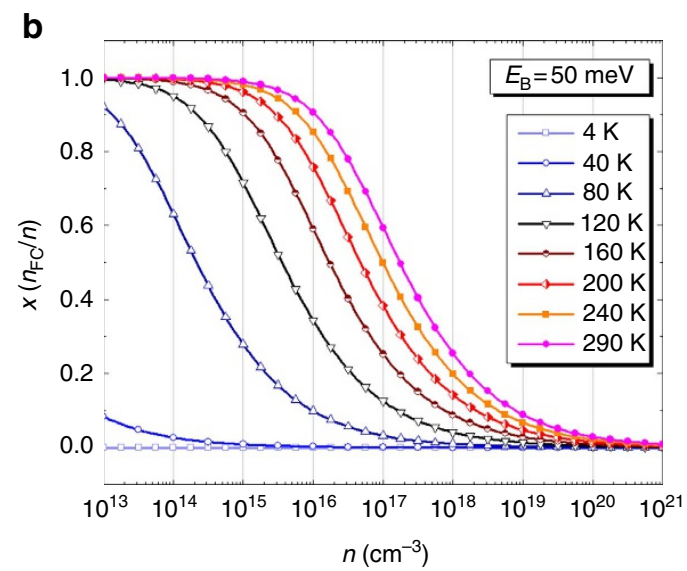

Figure 4 | Modelling of the fraction of free charges over the total photoexcitation. (a) Simulation of the free charge fraction over the total excitation density $\left(x=n_{\mathrm{FC}} / n\right)$ at thermal equilibrium according to equation (5) described in the text. For this simulation, we used an exciton binding energy of $(55 \pm 20) \mathrm{meV}$ and an exciton reduced mass, $\mu=0.15 \mathrm{~m}_{\mathrm{e}}$ (ref. 18). The shaded area represents $\mathrm{PV}$ operating conditions (PV regime). (b) Simultaion at different temperatures. With an exciton reduced mass $\mu=0.15 \mathrm{~m}_{\mathrm{e}}$ from ref. 18 and the estimated binding energy of 50 meV. 
value (in agreement with ref. 15) we can neglect exciton-exciton interaction, which only occurs for $n>10^{20} \mathrm{~cm}^{-3}$, because there is essentially no wavefunction overlap. Consequently, we can safely assume that the binding energy is independent of excitation density ${ }^{23,25}$. Within such an approximation, we can apply the law of mass action for Wannier-Mott excitons, as described by the Saha-Langmuir equation ${ }^{13,14}$. The fraction of free charges over the total density of excitation, $x$, for a 3D semiconductor can be expressed as $^{13}$ :

$$
\frac{x^{2}}{1-x}=\frac{1}{n}\left(\frac{2 \pi \mu k_{\mathrm{B}} T}{h^{2}}\right)^{3 / 2} e^{-\frac{E_{\mathrm{B}}}{k_{\mathrm{B}} T}}
$$

where $E_{\mathrm{B}}$ is the exciton binding energy, $\mu$ is the reduced mass of the exciton (approximated to $0.15 m_{\mathrm{e}}$ (ref. 18), see Supplementary Fig. 2 for modelling as a function of reduced exciton mass), $h$ is Planck's constant, $T$ is temperature and $n$ is the total density of excitation, $n=n_{\mathrm{FC}}+n_{\mathrm{exc}}$. The form of this equation is quite intuitive; the higher the temperature, the greater the ratio of free charge to excitons, but the higher the density of excitations, the lower the ratio of free charge due to the greater possibility for free electron-hole capture events. In Fig. 4a, we model the fraction of free charges over a wide range of total photoexcitation densities. Our key finding is that at RT, at excitation densities that may be realistic for operating solar cells $\left(n<10^{15} \mathrm{~cm}^{-3}\right)$, free charges represent the dominant population. The model shows that upon temperature reduction, the exciton population starts to increase, becoming dominant below $120 \mathrm{~K}$ (see Fig. 4b) ${ }^{13}$.

\section{Discussion}

We have identified that the exciton binding energy in the organolead mix-halide perovskite is $55 \pm 20 \mathrm{meV}$. This indicates that free charges are predominantly generated and are responsible for PV operation following light absorption, akin to a conventional inorganic semiconductor thin-film solar cell. This indicates that the role of the heterojunction in the perovskite solar cells is simply to enable selective charge collection, and not to facilitate exciton ionization. Notably, while perovskite cells are not excitonic in their operating principles, they still preserve a large absorption oscillator strength at the band edge, reminiscent of the underlying excitonic transition. This is a clear advantage in light harvesting near the band edge, enabling extremely thin-film solar cells to fully absorb light across the solar spectrum, and is in part responsible for the exceptional performance of the planar heterojunction perovskite solar cells ${ }^{9}$. Moreover, according to what we have shown, tuning the total density of photoexcitation allows for tuning of the nature of the primary photoexcitation at the thermodynamic equilibrium, that is, from free charges to excitons, thus opening up the way for application of these intriguing materials to a host of optoelectronic devices.

\section{Methods}

Sample preparation. Glass substrates were cleaned sequentially in $2 \%$ hallmanex detergent, acetone, propan-2-ol and oxygen plasma. The compact $\mathrm{TiO}_{2}$ layer $(\sim 50 \mathrm{~nm}$ ) was deposited by spin coating (at 2,000 r.p.m. for $60 \mathrm{~s}$ ) a mildly acidic solution of titanium isopropoxide in ethanol, followed by annealing at $500{ }^{\circ} \mathrm{C}$ for $30 \mathrm{~min}$. The $\sim 0.5 \mu \mathrm{m} \mathrm{TiO}$ and $\mathrm{Al}_{2} \mathrm{O}_{3}$ mesoporous layers were deposited by spin coating from stock solutions followed by sintering at $550{ }^{\circ} \mathrm{C}$ for $30 \mathrm{~min}$, as described previously ${ }^{3}$, with appropriate dilutions to give the thin mesoporous $\mathrm{Al}_{2} \mathrm{O}_{3}$ layer $(\sim 80 \mathrm{~nm})$.

The perovskite precursor solutions were prepared using methods described previously for the mixed halide ${ }^{3}$. In brief, the $\mathrm{CH}_{3} \mathrm{NH}_{3} \mathrm{PbI}_{3-\mathrm{x}} \mathrm{Cl}_{\mathrm{x}}$ or $\mathrm{CH}_{3} \mathrm{NH}_{3} \mathrm{PbI}_{3}$ precursor solutions were formed by dissolving methylammonium iodide and either lead (II) chloride (Sigma-Aldrich) or lead (II) iodide (Sigma-Aldrich) in anhydrous $\mathrm{N}, \mathrm{N}$-DimethylformamideDMF at a 3:1 molar ratio of $\mathrm{MAI}$ to $\mathrm{PbCl}_{2} / \mathrm{PbI}_{2}$, with final concentrations of $0.88 \mathrm{M}$ lead chloride/iodide and $2.64 \mathrm{M}$ methylammonium iodide. The perovskite layer was then formed by spin coating the precursor solutions directly on the glass substrate, compact layer or the mesoporous layer at 2,000 r.p.m. in air. The flat and thin meso samples were spin coated without dilution but mesoporous samples were spin coated from diluted (three parts in four) precursor solutions. After spin coating, the $\mathrm{CH}_{3} \mathrm{NH}_{3} \mathrm{PbI}_{3-\mathrm{x}} \mathrm{Cl}_{\mathrm{x}}$ films were annealed at $100{ }^{\circ} \mathrm{C}$ for $45 \mathrm{~min}$, and the $\mathrm{CH}_{3} \mathrm{NH}_{3} \mathrm{PbI}_{3}$ at $150^{\circ} \mathrm{C}$ for $15 \mathrm{~min}$. For the flat and thin mesoporous samples (that is, those without thick mesoporous layers), the perovskite films were sealed by spin coating a layer of the insulating polymer poly(methylmethacrylate) $\left(10 \mathrm{mg} \mathrm{ml}^{-1}, 1,000\right.$ r.p.m.; Sigma-Aldrich) on top in order to ensure air- and moisture-insensitivity.

Ultraviolet-visible absorption. Absorption measurements were performed using a spectrophotometer (Perkin Elmer Lambda 1050) and a continuous flow static exchange gas cryostat (Oxford Instruments Optistat CF). The cryostat consist of three chambers, one inside the other. The sample is housed inside the internal chamber filled with gaseous $\mathrm{He}$. The cryogenic liquid $(\mathrm{He})$ is fluxed inside the second chamber allowing temperature control of the He atmosphere of the sample chamber. Eventually, a third chamber is evacuated $\left(\sim 10^{-5}-10^{-6} \mathrm{mbar}\right)$ in order to assure thermal isolation from the external ambient.

\section{References}

1. Kim, H.-S. et al. Lead iodide perovskite sensitized all-solid-state submicron thin film mesoscopic solar cell with efficiency exceeding 9\%. Sci. Rep. 2, 591 (2012).

2. Burschka, J. et al. Sequential deposition route to high performance perovskite-sensitized solar cells. Nature 499, 316-320 (2013).

3. Kojima, A., Teshima, K., Shirai, Y. \& Miyasaka, T. Organometal halide perovskites as visible-light sensitizers for photovoltaic cells. J. Am. Chem. Soc. 131, 6050-6051 (2009).

4. Heo, J. H. et al. Efficient inorganic-organic hybrid heterojunction solar cells containing perovskite compound and polymeric hole conductors. Nat. Photon. 7, 487 (2013)

5. Lee, M. M., Teuscher, J., Miyasaka, T., Murakami, T. N. \& Snaith, H. J. Efficient hybrid solar cells based on meso-superstructured organometal halide perovskites. Science 338, 643-647 (2012)

6. Liu, M., Johnston, B. M. \& Snaith, J. H. Efficient planar heterojunction perovskite solar cells by vapour deposition. Nature 501, 395-398 (2013).

7. Ball, J. M., Lee, M. M., Hey, A. \& Snaith, H. J. Low-temperature processed mesosuperstructured to thin-film perovskite solar cells. Energy Environ. Sci. 6, 1739-1743 (2013)

8. Chopra, K. L., Paulson, P. D. \& Dutta, V. Thin-film solar cells: an overview. Prog. Photovolt. Res. Appl 12, 69-92 (2004).

9. Loi, M. A. \& Hummelen, J. C. Hybrid solar cells: perovskites under the sun. Nat. Mater. 12, 1087-1089 (2013).

10. Malinkiewicz, O. et al. Perovskite solar cells employing organic chargetransport layers. Nat. Photon. 8, 128-132 (2014).

11. Liu, D. \& Kelly, T. L. Perovskite solar cells with a planar heterojunction structure prepared using room-temperature solution processing techniques. Nat. Photon. 8, 133-138 (2014).

12. Yang-Yang et al. Low-temperature solution-processed perovskite solar cells with high efficiency and flexibility. ACS Nano 8, 1674-1680 (2014).

13. Cingolani, R. et al. Radiative recombination processes in wide-band-gap II-VI quantum wells: the interplay between excitons and free carriers. J. Opt. Soc. Am B 13, 1268 (1996).

14. Saha, M. N. On a physical theory of stellar spectra. Proc. R. Soc. Lond. A 99, 135-153 (1921).

15. Baikie, T. et al. Synthesis and crystal chemistry of the hybrid perovskite $\left(\mathrm{CH}_{3} \mathrm{NH}_{3}\right) \mathrm{PbI}_{3}$ for solid-state sensitised solar cell applications. J. Mater. Chem. A 1, 5628 (2013).

16. Ishiara, T., Takahashi, J. \& Goto, T. Optical properties due to electronic

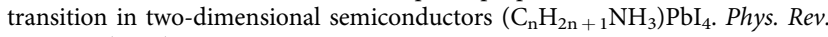
B 42, 17 (1990).

17. Hirasawa, M., Ishihara, T. \& Goto, T. Exciton features in 0-, 2-, and 3-dimensional networks of $\left[\mathrm{PbI}_{6}\right]^{4-}$-octahedra. J. Phys. Soc. Jpn 63, 3870-3879 (1994).

18. Tanaka, K. et al. Comparative study on the excitons in lead-halide-based perovskite-type crystals $\mathrm{CH} 3 \mathrm{NH} 3 \mathrm{PbBr} 3 \mathrm{CH} 3 \mathrm{NH} 3 \mathrm{PbI} 3$. Solid State Commun. 127, 619-623 (2003).

19. Varshni, Y. P. Temperature dependence of the energy gap in semiconductors Physica 34, 149-154 (1967).

20. Ashcroft, N. W. \& Mermin, N. D. Solid State Physics (Holt Rinehart \& Winston, 1976).

21. Wang, Y. et al. Optical transient bleaching of quantum-confined CdS clusters: the effects of surface-trapped electron-hole pairs. J. Chem. Phys. 92, 6927 (1990).

22. Lanzani, G. The Photophysics Behind Photovoltaics and Photonics (Wiley-VCH, Weinheim, 2012).

23. Viswanath, A. K., Lee, J. I., Kim, D., Lee, C. R. \& Leem, J. Y. Exciton-phonon interactions, exciton binding energy, and their importance in the realization of room-temperature semiconductor lasers based on GaN. Phys. Rev. B 58, 16333 (1998). 
24. Stranks, S. D. et al. Electron-hole diffusion lengths exceeding 1 micron in an organometal trihalide perovskite absorber. Science 342, 341-344 (2013).

25. Hendry, E., Koeberg, M. \& Bonn, M. Exciton and electron-hole plasma formation dynamics in ZnO. Phys. Rev. B 76, 045214 (2007).

\section{Acknowledgements}

We thank Dr F. Tassone and Prof R Cingolani for useful discussions on the photophysics of Wannier excitons; Dr J. Ball and G. Eperon for help with sample preparation; European Union Seventh Framework Programme [FP7/2007-2013] under grant agreement $n^{\circ} 604032$ of the MESO project and 'The Royal Society International Exchanges Scheme 2012/R2' for funding. H.J.S. thanks for funding the Engineering and Physical Sciences Research Council (EPSRC), the European Research Council (ERC-StG 2011 HYPER Project no. 279881), Oxford Photovoltaics Ltd. through a Nanotechnology KTN CASE award, and by a Royal Society Wolfson exchange grant. Dr S. Stranks thanks Worcester College, Oxford, for additional financial support.

\section{Author contributions}

The Ultraviolet-visible spectra were taken by V.D. and M.J.P.A. The simulations were run by V.D., G.G. and A.R.S.K. Samples were prepared by V.D., G.G., S.S. and M.L. All authors contributed to the data analysis and the manuscript preparation. A.P. and H.J.S. supervised the whole project.

\section{Additional information}

Supplementary Information accompanies this paper at http://www.nature.com/ naturecommunications

Competing financial interests: The authors declare no competing financial interests.

Reprints and permission information is available online at http://npg.nature.com/ reprintsandpermissions/

How to cite this article: D'Innocenzo, V. et al. Excitons versus free charges in organo-lead tri-halide perovskites. Nat. Commun. 5:3586 doi: 10.1038/ncomms4586 (2014). 